\title{
Influência da escolaridade e das condições de saúde no trabalho remunerado de idosos brasileiros
}

\author{
Influence of education and health conditions on paid work \\ of elderly Brazilians
}

Camila Menezes Sabino Castro (http://orcid.org/0000-0002-5383-9796) ${ }^{1}$

Maria Fernanda Lima Costa (http://orcid.org/0000-0002-3474-2980) ${ }^{1}$

Cibele Comini Cesar (http://orcid.org/0000-0003-2869-2291) ${ }^{2}$

Jorge Alexandre Barbosa Neves (http://orcid.org/0000-0003-4141-2282) ${ }^{3}$

Rosana Ferreira Sampaio (http://orcid.org/0000-0002-4775-9650) ${ }^{4}$

${ }^{1}$ Programa de PósGraduação em Saúde Coletiva, Instituto René Rachou, Fiocruz. Av. Augusto de Lima 911, Barro Preto. 30190-002 Belo Horizonte MG Brasil. camilamscastro@gmail.com

${ }^{2}$ Centro de

Desenvolvimento e

Planejamento Regional, Faculdade de Ciências

Econômicas, Universidade Federal de Minas Gerais (UFMG). Belo Horizonte MG Brasil.

${ }^{3}$ Departamento de Sociologia, Faculdade de Filosofia e Ciências Humanas, UFMG. Belo Horizonte MG Brasil. ${ }^{4}$ Programa de PósGraduação em Ciências da Reabilitação, UFMG. Belo Horizonte MG Brasil.

\begin{abstract}
The objective of this study was to determine associations between socio-demographic and health characteristics with paid work among elderly Brazilians. The analysis included 11,177 subjects aged 60 years and over from the National Health Survey conducted in 2013. The multivariate analysis was based on prevalence ratios and their respective 95\% confidence intervals estimated by Poisson regression. The participation of men in paid work was significantly higher (32.9\%) than women (14.4\%). For men and women, this participation decreased with increasing age and was higher among those with better schooling and good self-rated health. Among men, good self-rated health was associated with paid work $(P R=$ 1.63, 95\% CI, 1.23-2.15) only for those with less schooling $(<9$ years). Among women, good self-rated health revealed a positive association with paid work ( $P R=1.94,95 \%$ CI 1.32-2.84) for those with less schooling, and a negative association for those with better schooling $(R P=$ $0.54,95 \%$ CI 0.38-0.77). The results suggest that investments in schooling and improvements in health conditions can contribute to an increase in the longevity of the elderly in the labor market.

Key words Aging, Work, Educational status, Health
\end{abstract}

Resumo O objetivo do estudo foi determinar associações entre características sociodemográficas e de saúde com o trabalho remunerado entre idosos brasileiros. As análises incluíram 11.177 indivíduos com 60 anos ou mais, da Pesquisa Nacional de Saúde, conduzida em 2013. A análise multivariada foi baseada em razões de prevalência e seus respectivos intervalos de confiança de 95\%, estimados pela regressão de Poisson. A participação dos homens no trabalho remunerado foi significativamente mais alta (32,9\%) em comparação às mulheres (14,4\%). Para homens e mulheres, essa participação diminuiu com o aumento de idade e foi maior entre aqueles com maior escolaridade e boa autoavaliação da saúde. Entre os homens, a boa autoavaliação da saúde apresentou associação com o trabalho remunerado $(R P=1,63$; IC95\%:1,23-2,15) somente para aqueles com escolaridade mais baixa (<9 anos). Entre as mulheres, a boa autoavaliação da saúde apresentou associação positiva com o trabalho remunerado ( $R P$ = 1,94; IC95\%:1,32-2,84) para aquelas com escolaridade mais baixa, e associação negativa para aquelas com escolaridade mais alta $(R P=0,54$; IC95\%:0,38-0,77). Os resultados sugerem que investimentos na escolaridade e melhorias nas condições de saúde podem contribuir para aumentar a longevidade dos idosos no mercado de trabalho. Palavras-chave Envelhecimento, Trabalho, Escolaridade, Saúde 


\section{Introdução}

O envelhecimento da população é um fenômeno global e o Brasil é um dos países onde esse processo ocorre com maior velocidade ${ }^{1,2}$. Países de alta renda vivenciaram as mudanças demográficas de forma gradual, acompanhado de progresso econômico, melhoria das condições de vida e saúde da população e um amplo sistema de proteção social $^{3}$. Por outro lado, países de média e baixa renda, como o Brasil, ainda vivem um momento no qual a maior parte da sua população está em idade de trabalhar (20-64 anos). Essa janela de oportunidades permanecerá até por volta de 2030. No entanto, a partir de 2035 , a população apresentará uma diminuição do seu contingente, inclusive da força de trabalho e, a partir de 2045, apenas a população brasileira com 60 anos ou mais poderá ter um incremento positivo, levando a uma estrutura etária super-envelhecida ${ }^{4}$.

A Organização Mundial da Saúde chama a atenção para a necessidade da promoção do envelhecimento ativo, sem o qual haverá risco de quebra dos sistemas sociais e de saúde em função do envelhecimento da população. $\mathrm{O}$ envelhecimento ativo baseia-se no tripé saúde, participação e segurança. Essa proposta traz um novo paradigma, reconhecendo a necessidade de apoiar medidas que possibilitem às pessoas mais velhas participar e contribuir ativamente na sociedade ${ }^{5}$. Nessa perspectiva, o trabalho nas faixas etárias mais velhas assume grande relevância. A inserção dos idosos no mercado de trabalho é determinada por melhorias na educação, nas condições de saúde e implementação de políticas que permitam aos indivíduos permanecerem trabalhando em idades avançadas ${ }^{1}$.

Embora com diferentes dinâmicas temporais e magnitude, a participação dos idosos no mercado de trabalho está aumentando em todo o mundo. No Reino Unido a proporção de pessoas com 50-64 anos de idade que trabalham subiu de $57 \%$, em 1995, para 69\%, em 2015, e a proporção correspondente para pessoas com 65 anos ou mais dobrou, passando de 5 para $10 \%$, no mesmo período $^{6}$. Na Austrália, a proporção de pessoas com 60-64 anos que participam do mercado de trabalho aumentou de $32 \%$, em 2000 , para $54 \%$, em 2014 ${ }^{7}$. Nos Estados Unidos, em 2015, cerca de um terço da força de trabalho era composta de pessoas com 50 anos e mais e as projeções são de que, em 2020, o número de trabalhadores com 55 anos ou mais aumente $20 \%{ }^{8}$. No Brasil, as projeções são de que, em 2020, pelo menos 13\% da população trabalhadora tenha mais de 60 anos, contra 9\%, em 19889. Dados da Pesquisa Nacional de Amostra por Domicílio ${ }^{10}$ mostraram um discreto aumento da participação dos idosos na força de trabalho. No segundo trimestre de 2017, brasileiros com 60 anos ou mais de idade correspondiam a 7,4\% das pessoas ocupadas, enquanto, em 2012, essa proporção era de 6,3\%.

De modo geral, os idosos permanecem trabalhando porque gostam dos seus empregos ou porque não têm condições econômicas para se aposentar ${ }^{6,11}$. Tanto nos países de alta, quanto nos de média renda a participação de idosos no mercado de trabalho é influenciada por vários fatores, tais como: sexo, idade, raça/cor, escolaridade, composição familiar, renda, condições de saúde, características da ocupação e fatores contextuais (participação social, mercado de trabalho e cobertura do sistema previdenciário) ${ }^{12-26}$. Ressalta-se que há uma importante diferença de gênero nessa relação. A proporção de homens no mercado de trabalho é maior que a das mulheres. Pesquisas mostram que a participação no trabalho remunerado entre as mulheres não depende somente da demanda do mercado ou das suas qualificações e condições de saúde, mas decorre também de uma articulação complexa de características pessoais e familiares, como presença de filhos e posição na família ${ }^{27}$.

Para o Brasil, estimular a participação das pessoas mais velhas na força de trabalho é uma questão desafiadora. Segundo Wajnman et al. ${ }^{9}$, os brasileiros que mais participam do trabalho são os mais dependentes financeiramente, ou seja, homens, negros, chefes de família com baixa renda e os não aposentados. Entretanto, à medida que envelhecem, as melhores chances de permanecer trabalhando pertencem aos mais qualificados, com escolaridade mais alta e, sobretudo, que não estão inseridos em atividades manuais. A literatura consultada apresenta poucos estudos que examinaram a influência da escolaridade na associação entre condições de saúde e trabalho remunerado entre idosos no Brasil e nenhum foi conduzido em amostra nacional representativa da população idosa. No município de Belo Horizonte observouse que a participação no trabalho remunerado era maior entre homens mais velhos com pior autoavaliação da saúde, tanto entre aqueles com baixa como mais alta escolaridade. Entre as mulheres, a participação no trabalho diminuiu drasticamente entre aquelas com pior autoavaliação da saúde e nível mais baixo de escolaridade ${ }^{16}$.

O presente estudo tem como objetivo determinar as condições sociodemográficas e de saúde associadas ao trabalho remunerado em amostra 
Trata-se de um estudo transversal, com dados da Pesquisa Nacional de Saúde (PNS 2013), cuja amostra foi delineada para representar a população brasileira com 18 anos ou mais. A pesquisa é de base domiciliar, de âmbito nacional, realizada pelo Instituto Brasileiro de Geografia e Estatística (IBGE), em parceria com o Ministério da Saúde (MS) e a Fundação Instituto Oswaldo Cruz (Fio-

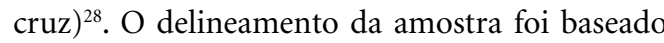
em três estágios de seleção. Os setores censitários formaram as unidades primárias de seleção, os domicílios as unidades secundárias e um morador do domicílio com 18 anos ou mais de idade a unidade terciária ${ }^{29}$. A coleta de dados foi baseada em três questionários, quais sejam: domiciliar, individual respondido por todos os moradores e individual, respondido por uma amostra probabilística dos moradores no domicílio com 18 anos ou mais. Para a presente análise, foram selecionados todos aqueles com 60 anos ou mais, que faziam parte da amostra de moradores do domicílio ( $\mathrm{N}=11.177)$.

A variável dependente do estudo foi trabalho remunerado nos últimos sete dias. Trabalho remunerado foi considerado como exercício de qualquer atividade com remuneração em dinheiro, produtos, mercadorias ou benefícios. Essa variável incluiu assalariados, patrões, trabalho por conta-própria e autônomo, trabalho por produção e biscate, pessoas afastadas por motivo de férias, licença por doença, entre outros. Todas as outras condições, quais sejam aposentados, pedintes, estudantes, donas de casa que se dedicam exclusivamente aos afazeres domésticos, indivíduos que trabalham sem remuneração ou se dedicam a trabalho voluntário e aqueles que viviam exclusivamente de renda ou ajuda de parentes e/ ou conhecidos foram incluídos na categoria não trabalho.

As variáveis independentes incluíram características sociodemográficas e condições de saúde. As variáveis sociodemográficas consideradas foram idade na data da entrevista, escolaridade (número de anos completos de estudo, categorizada como 0-8 anos vs 9 anos ou mais), raça/cor, situação conjugal (vive ou não com o(a) cônjuge ou o(a) companheiro(a)), posição de responsável pelo domicílio e local de residência (urbano ou rural). As condições de saúde foram divididas em saúde geral, número de doenças e condições crônicas e limitação dos sentidos. A saúde geral foi determinada pela autoavaliação da saúde, definida pela resposta à pergunta Em geral, como $o$ (a) $\operatorname{sr}($ a) avalia a sua saúde?. Nesta análise, as respostas foram categorizadas como boa (regular/boa/muito boa) e ruim (ruim/muito ruim). As doenças e condições crônicas foram definidas pela resposta à pergunta Algum médico disse que $o$ (a) senhor(a) tinha "tal doença"?. As doenças consideradas foram hipertensão, diabetes, doença do coração, artrite, problema crônico de coluna, distúrbios osteomusculares relacionados ao trabalho (DORT) e depressão. A limitação dos sentidos foi definida pela pergunta Em geral, que grau de dificuldade o(a) sr(a) tem para (...)?, considerando audição e visão, com cinco possibilidades de resposta (nenhuma, leve, média, intensa e não consegue). Nesta variável as respostas foram categorizadas como sim (leve, média, intensa e não consegue) vs não (nenhum). Além das variáveis acima mencionadas, para fins de descrição da amostra, foram consideradas a posição na ocupação entre os que informaram ter trabalho remunerado nos últimos sete dias, horas semanais de trabalho e renda mensal do trabalho.

A análise não ajustada foi baseada no teste qui-quadrado de Pearson para examinar diferenças de frequências entre gêneros na descrição das características dos participantes do estudo. A análise multivariada foi baseada em razões de prevalência e respectivos intervalos de confiança de 95\% (IC 95\%) estimados pela regressão de Poisson robusta. A análise multivariada foi baseada em dois modelos. O primeiro incluiu as características sociodemográficas. No segundo modelo, foram adicionadas as variáveis condições de saúde. Todas as análises foram realizadas separadamente para homens e mulheres. Foi usado o pacote estatístico Stata13.0 (College Station, Texas, USA), levando-se em conta os procedimentos para amostras complexas, de forma a considerar os parâmetros amostrais e o peso dos indivíduos nas análises. Adicionalmente, foi utilizado o comando subpop para seleção dos indivíduos com 60 anos e mais de idade.

A PNS foi aprovada pela Comissão Nacional de Ética em Pesquisa - CONEP.

\section{Resultados}

Participaram do estudo 11.177 idosos de ambos os sexos. A média de idade dos participantes foi 
69,8 anos e a proporção de mulheres foi igual a $56,4 \%$. Como mostrado na Tabela 1 , a escolaridade inferior a 8 anos predominou amplamente $(77,7 \%)$. A participação no trabalho remunerado foi informada por $22,4 \%$ dos participantes, com importante diferença entre os gêneros (32,9\% entre homens e 14,4\% entre mulheres; $\mathrm{p}<0,001)$. O trabalho por conta própria predominou $(51,3 \%$ entre os que tinham trabalho remunerado), tanto entre os homens $(54,6 \%)$ quanto entre as mulheres $(44,9 \%)$. Para os ho- mens, o trabalho no setor privado ocupou a $2^{\text {a }}$ posição $(26,0 \%)$, ao passo que para as mulheres essa posição foi ocupada pelo trabalho doméstico $(19,7 \%)$. A média semanal de horas de trabalho foi maior entre os homens (39,3 horas) em comparação às mulheres (32,1 horas). A remuneração mensal do trabalho foi 1,5 vezes maior entre os homens $(\mathrm{R} \$ 2.538,41)$ em comparação às mulheres ( $\mathrm{R} \$ 1.599,15)$.

Na Tabela 2 estão apresentados os resultados da análise multivariada das associações entre ca-

Tabela 1. Características sociodemográficas, de saúde e de trabalho dos idosos brasileiros, segundo o gênero

(Brasil, 2013).

\begin{tabular}{|c|c|c|c|c|}
\hline Variáveis & Total $(\%)^{\mathrm{a}}$ & Homens $(\%)^{a}$ & Mulheres $(\%)^{a}$ & $\begin{array}{c}\text { Valor } \\
p^{b}\end{array}$ \\
\hline \multicolumn{5}{|l|}{ Características sociodemográficas } \\
\hline \multicolumn{5}{|l|}{ Idade } \\
\hline $60-64$ & 31,9 & 31,5 & 32,2 & 0,554 \\
\hline $65-75$ & 42,8 & 43,8 & 42,0 & \\
\hline 75 e mais & 25,3 & 24,7 & 25,8 & \\
\hline Escolaridade (8 anos ou menos) & 77,7 & 77,2 & 78,1 & 0,536 \\
\hline \multicolumn{5}{|l|}{ Raça/cor } \\
\hline Branca & 53,7 & 52,7 & 54,3 & 0,619 \\
\hline Preta/parda & 44,8 & 45,7 & 44,2 & \\
\hline Amarela/Indígena & 1,5 & 1,6 & 1,4 & \\
\hline Possui cônjuge & 57,4 & 76,1 & 42,9 & $<0,001$ \\
\hline Responsável pelo domicílio & 64,7 & 78,1 & 54,4 & $<0,001$ \\
\hline Residência rural & 14,8 & 17,5 & 12,7 & $<0,001$ \\
\hline \multicolumn{5}{|l|}{ Características de saúde } \\
\hline Autoavaliação da saúde boa & 87,9 & 89,1 & 87,0 & 0,037 \\
\hline \multicolumn{5}{|l|}{ Número de doenças físicas ${ }^{c}$} \\
\hline Nenhuma & 27,6 & 33,4 & 23,2 & $<0,001$ \\
\hline Pelo menos uma & 32,8 & 34,2 & 31,8 & \\
\hline Duas ou mais & 39,6 & 21,4 & 45,1 & \\
\hline Limitação dos sentidos ${ }^{\mathrm{d}}$ & 68,7 & 68,8 & 68,7 & 0,931 \\
\hline Total & 11.177 & 4.555 & 6.622 & \\
\hline \multicolumn{5}{|l|}{ Características de trabalho ${ }^{\mathrm{e}}$} \\
\hline Trabalho remunerado & 22,4 & 32,9 & 14,4 & $<0,001$ \\
\hline \multicolumn{5}{|l|}{$\begin{array}{l}\text { Posição na ocupação entre os que } \\
\text { informaram ter trabalho remunerado }\end{array}$} \\
\hline Trabalhador doméstico & 8,2 & 1,8 & 19,7 & \\
\hline Militar & 0,1 & 0,2 & 0,1 & $<0,001$ \\
\hline Empregado setor privado & 21,2 & 26,0 & 12,6 & \\
\hline Empregado setor público & 12,2 & 9,7 & 16,8 & \\
\hline Empregador & 7,0 & 7,7 & 5,9 & \\
\hline Conta-própria & 51,3 & 54,6 & 44,9 & \\
\hline $\begin{array}{l}\text { Horas semanais de trabalho, média } \\
\text { (DP) }\end{array}$ & $36,9(15,56)$ & $39,3(14,72)$ & $32,1(16,05)$ & $<0,001$ \\
\hline $\begin{array}{l}\text { Renda mensal do trabalho em reais, } \\
\text { média (DP) }\end{array}$ & $2.457,71(2.230,10)$ & $2.538,41(10.375,12)$ & $1.599,15(2.940,52)$ & $<0,001$ \\
\hline Total & 5.579 & 3.743 & 1.836 & \\
\hline
\end{tabular}


Tabela 2. Análise multivariada da associação entre trabalho remunerado, características sociodemográficas e de saúde, segundo o gênero (Brasil, 2013).

\begin{tabular}{|c|c|c|c|c|}
\hline \multirow[b]{2}{*}{ Variáveis } & \multicolumn{2}{|c|}{ Homens } & \multicolumn{2}{|c|}{ Mulheres } \\
\hline & $\begin{array}{c}\text { Modelo 1 } \\
\text { Sócio- } \\
\text { demográfico } \\
\text { RP (IC95\%) }\end{array}$ & $\begin{array}{c}\text { Modelo 2 } \\
\text { Condições de } \\
\text { saúde } \\
\text { RP }(\text { IC95\%) } \\
\end{array}$ & $\begin{array}{c}\text { Modelo 1 } \\
\text { Sócio- } \\
\text { demográfico } \\
\text { RP (IC95\%) }\end{array}$ & $\begin{array}{c}\text { Modelo } \\
2 \text { Condições de } \\
\text { saúde } \\
\text { RP }(\text { IC95\%) } \\
\end{array}$ \\
\hline \multicolumn{5}{|l|}{ Idade $60-64$ anos $v s$} \\
\hline $65-75$ & $0,54(0,46-0,62)$ & $0,54(0,47-0,63)$ & $0,34(0,27-0,42)$ & $0,34(0,27-0,42)$ \\
\hline 75 e mais & $0,27(0,21-0,36)$ & $0,28(0,22-0,37)$ & $0,09(0,06-0,14)$ & $0,09(0,06-0,14)$ \\
\hline Escolaridade 0-8 vs 9+ anos & $1,31(1,13-1,51)$ & $1,28(1,10-1,48)$ & $1,73(1,39-2,16)$ & $1,72(1,37-2,17)$ \\
\hline \multicolumn{5}{|l|}{ Raça/cor branca $v s$} \\
\hline Preta/parda & $1,02(0,89-1,16)$ & $1,04(0,91-1,18)$ & $1,06(0,87-1,29)$ & $1,07(0,88-1,31)$ \\
\hline Amarela/Indígena & $1,07(0,61-1,90)$ & $1,05(0,60-1,86)$ & $1,06(0,55-2,06)$ & $1,08(0,56-2,09)$ \\
\hline Possui cônjuge & $1,30(1,10-1,53)$ & $1,28(1,10-1,51)$ & $0,85(0,67-1,08)$ & $0,86(0,68-1,09)$ \\
\hline Responsável pelo domicílio & $1,22(1,00-1,48)$ & $1,20(0,98-1,47)$ & $1,08(0,83-1,41)$ & $1,08(0,83-1,40)$ \\
\hline Residência rural & $1,22(1,05-1,42)$ & $1,24(1,07-1,44)$ & $0,82(0,60-1,13)$ & $0,83(0,60-1,14)$ \\
\hline $\begin{array}{l}\text { Boa autoavaliação da saúde } v s \\
\text { ruim/muito ruim }\end{array}$ & - & $1,58(1,23-2,04)$ & - & $1,28(0,92-1,79)$ \\
\hline \multicolumn{5}{|l|}{ Nenhuma doença crônica $^{\mathrm{b}}$} \\
\hline Pelos menos uma & - & $1,01(0,87-1,17)$ & - & $1,01(0,81-1,26)$ \\
\hline Duas ou mais & & $0,93(0,77-1,13)$ & & $1,07(0,84-1,35)$ \\
\hline Limitação dos sentidos $^{c}$ & - & $0,92(0,80-1,06)$ & - & $1,12(0,90-1,40)$ \\
\hline $\begin{array}{l}\text { IC95\%: intervalos de } 95 \% \text { de confian } \\
\text { ajustado por idade, escolaridade, raça } \\
2 \text { - modelo } 1 \text { acrescido da autoavalia } \\
\text { da regressão de Poisson; }{ }^{b} \text { Diagnóstic }\end{array}$ & saúde boa, número & doenças crônicas e 1 & $\begin{array}{l}\text { le entrevistados) }=1 \\
\text { domicílio e residên } \\
\text { hitação dos sentidos. }\end{array}$ & $\begin{array}{l}\text { 177. Modelo } 1 \text { - } \\
\text { a urbana. Modelo } \\
\text { Estimadas por meio } \\
\text { rônico na coluna, }\end{array}$ \\
\hline
\end{tabular}

racterísticas sociodemográficas e de saúde e trabalho remunerado, segundo o gênero. No modelo completamente ajustado, associação positiva e estatisticamente significante $(\mathrm{p}<0,05)$ foi observada para escolaridade superior a 8 anos para ambos os gêneros. Associação negativa tanto para homens quanto para as mulheres foram observadas para a idade. Somente entre os homens, associações positivas também foram observadas para residência com o cônjuge, residência em área rural e boa auto-avaliação da saúde.

$\mathrm{Na}$ Tabela 3 estão apresentados os resultados da análise multivariada das associações entre características sociodemográficas e de saúde e trabalho remunerado, segundo o gênero e a escolaridade. Entre homens e mulheres, as associações negativas com a idade permaneceram para os idosos com baixa e alta escolaridade. A associação positiva com residência com o cônjuge e com residência rural permaneceu entre os homens com baixa escolaridade, mas não entre aqueles com escolaridade mais alta. Importantes diferenças foram observadas com referência à autoavaliação da saúde. Entre homens com baixa escolaridade, a boa autoavaliação da saúde apresentou associa- ção estatisticamente significante com o trabalho remunerado ( $\mathrm{RP}=1,63$; IC 95\% 1,23-2,15), mas entre aqueles com escolaridade mais alta a associação não foi estatisticamente significante ( $\mathrm{RP}=$ 1,19; IC 95\% 0,71-1,99). Entre as mulheres, associação positiva com o trabalho remunerado foi observada entre aquelas com escolaridade mais baixa ( $\mathrm{RP}=1,94$; IC 95\% 1,32-2,84) e associação negativa para aquelas com escolaridade mais alta $(\mathrm{RP}=0,54$; IC 95\% 0,38-0,77).

\section{Discussão}

Os resultados da presente análise, baseada em amostra nacional de idosos brasileiros, mostraram que existem semelhanças e diferenças nos fatores associados à participação de homens e mulheres idosos no trabalho remunerado. Para ambos os gêneros, a participação no trabalho remunerado diminuiu com a idade e foi mais alta entre aqueles com melhor escolaridade e boa autoavaliação da saúde, independentemente de outros fatores relevantes. Os resultados também mostraram que alguns fatores associados a essa 
Tabela 3. Análise multivariada da associação entre trabalho remunerado, características sociodemográficas e de saúde, segundo o nível de escolaridade e o gênero (Brasil, 2013).

\begin{tabular}{|c|c|c|c|c|}
\hline \multirow[b]{2}{*}{ Variáveis } & \multicolumn{2}{|c|}{ Homens } & \multicolumn{2}{|c|}{ Mulheres } \\
\hline & $\begin{array}{c}\text { Escolaridade baixa } \\
\text { RP (IC95\%) }\end{array}$ & $\begin{array}{l}\text { Escolaridade alta } \\
\text { RP (IC95\%) }\end{array}$ & $\begin{array}{c}\text { Escolaridade baixa } \\
\text { RP (IC95\%) }\end{array}$ & $\begin{array}{c}\text { Escolaridade alta } \\
\text { RP }(\text { IC95\% })^{\mathrm{a}}\end{array}$ \\
\hline \multicolumn{5}{|l|}{ Idade $60-64$ anos $v s$} \\
\hline $65-75$ & $0,54(0,45-0,65)$ & $0,56(0,44-0,71)$ & $0,34(0,25-0,44)$ & $0,36(0,26-0,49)$ \\
\hline 75 e mais & $0,29(0,21-0,39)$ & $0,27(0,15-0,46)$ & $0,10(0,06-0,16)$ & $0,08(0,04-0,16)$ \\
\hline \multicolumn{5}{|l|}{ Raça/cor branca $v s$} \\
\hline Preta/parda & $1,02(0,87-1,20)$ & $1,06(0,84-1,33)$ & $1,12(0,85-1,46)$ & $1,02(0,81-1,27)$ \\
\hline Amarela/Indígena & $0,71(0,28-1,84)$ & $1,28(0,66-2,50)$ & $1,24(0,51-2,99)$ & $0,92(0,36-2,36)$ \\
\hline Possui cônjuge & $1,30(1,06-1,60)$ & $1,21(0,96-1,52)$ & $0,97(0,72-1,30)$ & $0,73(0,49-1,09)$ \\
\hline Responsável pelo domicílio & $1,26(0,98-1,62)$ & $1,11(0,82-1,51)$ & $1,19(0,88-1,61)$ & $0,89(0,56-1,43)$ \\
\hline Residência rural & $1,23(1,05-1,44)$ & $1,15(0,72-1,85)$ & $0,87(0,62-1,23)$ & $0,49(0,20-1,17)$ \\
\hline $\begin{array}{l}\text { Boa autoavaliação da saúde } \\
v s \text { ruim/muito ruim }\end{array}$ & $1,63(1,23-2,15)$ & $1,19(0,71-1,99)$ & $1,94(1,32-2,84)$ & $0,54(0,38-0,77)$ \\
\hline \multicolumn{5}{|l|}{ Nenhuma doença crônica ${ }^{\mathrm{b}}$} \\
\hline Pelos menos uma & $0,98(0,82-1,18)$ & $1,07(0,85-1,34)$ & $0,90(0,66-1,25)$ & $1,14(0,84-1,55)$ \\
\hline Duas ou mais & $0,90(0,71-1,13)$ & $1,00(0,71-1,40)$ & $1,12(0,82-1,53)$ & $0,89(0,63-1,25)$ \\
\hline Limitação dos sentidos ${ }^{\mathfrak{c}}$ & $0,86(0,72-1,03)$ & $1,09(0,86-1,38)$ & $1,24(0,94-1,63)$ & $0,92(0,65-1,31)$ \\
\hline
\end{tabular}

participação foram modificados pelo nível de escolaridade, em particular a condição geral da saúde.

A proporção mais elevada de homens idosos no mercado de trabalho, observada nesta análise, está em consonância com estudos realizados em outros países, como a Austrália ${ }^{12}$, a Itália e a Espa$\mathrm{nha}^{24} \mathrm{e}$ em alguns municípios brasileiros, como Belo Horizonte ${ }^{16}$ e São Paulo ${ }^{25}$. Essas diferenças podem ser explicadas, pelo menos em parte, pelas situações adversas que as atuais coortes de idosas enfrentaram ao longo das suas vidas, como baixo nível educacional, menor investimento no desenvolvimento de habilidades, menos oportunidades econômicas e menos direito à propriedade ${ }^{30}$. Além disso, em quase todos os países, as mulheres têm maior propensão a exercer atividades menos qualificadas, ter emprego familiar assalariado ou não remunerado, a participar do mercado de trabalho informal, a gerenciar empresas menores e a se concentrar nos setores menos lucrativos ${ }^{31}$. Os resultados reforçam essas observações por mostrar que o trabalho doméstico foi a $2^{\mathrm{a}}$ ocupação mais frequente entre as mulheres, ao passo que entre os homens essa posição foi ocupada pelo trabalho no setor privado. Adicionalmente, é importante destacar que a remuneração pelo trabalho entre os homens foi 50\% maior que a das mulheres, reforçando as disparidades entre os gêneros ${ }^{32}$.
A redução do trabalho remunerado com o aumento da idade, como observado para ambos os gêneros na presente análise, é esperada, uma vez que as condições de saúde pioram e a limitação funcional aumenta exponencialmente com a idade ${ }^{33}$. Entretanto, existem também dificuldades para a manutenção ou obtenção do emprego formal entre idosos. Pesquisas anteriores mostram que os idosos são menos propensos a encontrar novo emprego, comparado aos mais jovens e quando encontram são, frequentemente, confrontados com cortes salariais ${ }^{17,21,34,35}$. Segundo West et al. ${ }^{21}$ a duração média de desemprego para americanos acima de 55 anos é de 35,5 semanas, em comparação a 23,3 semanas para os jovens (16-24 anos). Além disso, diversos estudos têm mostrado que o trabalho autônomo tende a aumentar com a idade ${ }^{17,35,36}$. Nesse sentido, é importante salientar que os resultados deste estudo mostraram que o trabalho autônomo foi a ocupação mais frequente entre idosos de ambos os gêneros.

Nesta análise, a residência com o cônjuge e a residência em área rural mostraram associação positiva com o trabalho remunerado entre os homens, mas não entre as mulheres. Na análise estratificada pelo nível de escolaridade, as associações acima mencionadas persistiram somente entre aqueles com escolaridade mais baixa. Uma hipótese plausível para a associação relativa à re- 
sidência com o cônjuge é o fato de que os homens com menor escolaridade sejam responsáveis pela família, tendo que prover o sustento do $\operatorname{lar}^{27}$. Por sua vez, homens idosos com baixa escolaridade que vivem em zona rural são mais propensos ao trabalho remunerado, provavelmente pelo fato desses indivíduos apresentarem ganhos mais baixos ao longo da vida, comparados aos seus correspondentes que moram em zona urbana e/ou às altas taxas de migração rural pelos trabalhadores mais jovens ${ }^{12}$.

Um achado consistente na literatura, tanto em países de alta quanto de média renda é o de que o nível de escolaridade aumenta a participação dos idosos na força de trabalho ${ }^{12,37}$. Por exemplo, na Austrália, verificou-se entre as mulheres de 55-64 anos, um ano adicional de escolaridade aumenta a probabilidade de participação na força de trabalho em $5,1 \%$, enquanto entre os homens da mesma faixa etária, o aumento foi de $4,5 \%{ }^{37}$. Os resultados são consistentes com essas observações. A escolaridade foi um dos fatores mais fortemente associados à participação dos idosos e idosas no mercado de trabalho remunerado.

Outro achado relevante foi de que a autoavaliação da saúde, mas não o número de doenças crônicas e a limitação dos sentidos, apresentou associação com ter trabalho remunerado. A autoavaliação da saúde é um indicador multidimensional, que reflete a saúde física, mental e social do indivíduo, consistindo em uma expressão do reconhecimento do indivíduo dos seus sintomas, diagnósticos médicos e/ou diminuição da funcionalidade $^{38,39}$. Um estudo baseado na coorte de idosos de Bambuí mostrou que a avaliação da autoavaliação da saúde no inquérito da linha de base apresentava valor preditivo para a mortalidade, ao longo dos 10 anos subsequentes, semelhante à de 11 medidas objetivas da condição da saúde ${ }^{39}$. Estudo anterior com adultos participantes do Inquérito Mundial de Saúde no Brasil mostrou que, entre os homens, os fatores que mais contribuíram para a pior autoavaliação da saúde, além de posse de bens materiais, foram os fatores relacionados ao trabalho, como trabalho manual, desemprego e aposentadoria por inva- lidez ${ }^{40}$. Outro estudo, conduzido entre adultos brasileiros, mostrou que "não ter trabalho" estava associado à pior autoavaliação da saúde entre homens e mulheres, mas que a magnitude da associação era maior entre os homens ${ }^{41}$. Os resultados do estudo acrescentam a esses achados por mostrar que a boa autoavaliação da saúde apresentou associação positiva com ter trabalho remunerado entre homens e mulheres idosos com nível mais baixo de escolaridade, mas não entre aqueles com nível mais alto. Uma possível explicação para essas diferenças é a de que indivíduos com maior nível de escolaridade estão em ocupações que demandam maior exigência mental, como atividades nas áreas da educação, saúde e bem-estar social, humanidades e artes, enquanto seus correspondentes com menor nível de escolaridade estão em ocupações com maior exigência física $^{22,27}$.

As pesquisas brasileiras sobre trabalho e envelhecimento ainda são escassas e em sua maioria foram conduzidas utilizando dados municipais ou regionais ${ }^{16,22,25,26,42}$. Portanto, uma vantagem da presente investigação é sua grande base populacional, com representatividade nacional. Entre as limitações estão a natureza transversal da pesquisa, que não permite estabelecer relações temporais das variáveis observadas. $\mathrm{O}$ efeito do trabalhador sadio é um viés a ser considerado ao analisar a associação positiva entre participação no trabalho remunerado e saúde, pois idosos mais saudáveis apresentam maior probabilidade de permanecer trabalhando.

Concluindo, os resultados sugerem que investimentos na escolaridade e melhorias nas condições de saúde podem contribuir para aumentar a longevidade dos idosos no mercado de trabalho. Os resultados também mostram importantes e persistentes disparidades entre os gêneros de forma que as idosas, em comparação aos idosos, tendem a ter um trabalho menos qualificado e com menor remuneração. A necessidade de prolongar a vida no trabalho tem sido cada vez mais reconhecida e para isto faz-se necessário investimentos e políticas para assegurar que as pessoas de todas as idades permaneçam ativas. 


\section{Colaboradores}

CMS Castro, MFL Costa, CC César, JAB Neves e RF Sampaio trabalharam na concepção, análise e interpretação dos resultados, preparação e redação do manuscrito e revisão crítica do conteúdo. Todos os autores leram e aprovaram a versão final do manuscrito.

\section{Agradecimentos}

Os autores gostariam de agradecer o financiamento proporcionado pelo Conselho Nacional de Desenvolvimento Científico e Tecnológico $(\mathrm{CNPq})$, pela Fundação de Amparo à Pesquisa de Minas Gerais (Fapemig) e pela Fundação Oswaldo Cruz (Fiocruz).

\section{Referências}

1. United Nations (UN). Department of Economic and Social Affairs, Population Division. World Population Ageing 2015. [acessado 2016 Jul 29]. Disponível em: http://esa.un.org/wpp

2. Wong LLR, Carvalho JA. O rápido processo de envelhecimento populacional do Brasil: sérios desafios para as políticas públicas. Rev Bras Est Pop 2006; 23:5-26.

3. Kreling NH. O envelhecimento do trabalhador impõe novos desafios às políticas públicas. Apresentado na XVII Encontro Nacional de estudos Populacionais, ABEP. Caxambu, Brasil, 2010. [acessado 2016 Ago 4]. Disponível em: http://www.abep.org.br

4. Camarano AA. Perspectivas de crescimento da população brasileira e algumas implicações. In: Camarano AA, organizador. Novo regime demográfico: uma nova relação entre população e desenvolvimento? Rio de Janeiro: IPEA; 2014. p. 177-210.

5. World Health Organization (WHO). Active ageing: a policy framework. Geneva: WHO; 2002.

6. Programa das Nações Unidas para o Desenvolvimento (PNUD). Relatório do desenvolvimento humano 2015: O trabalho como motor do desenvolvimento humano. [acessado 2016 Ago 2]. Disponível em: http://www.undp.org

7. Zacher H, Griffin B. Work, aging, and retirement in Australia: introduction to the special issue. Work, aging and retirement $2015 ; 1(2): 129-132$.

8. Wargo-Sugleris M. Job Satisfaction, Work environment, and successful aging: determinants to remain in older acute care nurses [tese]. Los Angeles: University of California; 2015.

9. Wajnman S, Oliveira AMHC, Oliveira EL. Os idosos no mercado de trabalho: tendências e consequências. In: Camarano AA, organizadora. Os novos idosos brasileiros muito além dos 60? Rio de Janeiro: IPEA; 2004. p. 453-479.

10. Instituto Brasileiro de Geografia e Estatística (IBGE). Indicadores IBGE. Pesquisa Nacional por Amostra de Domicílios Contínua - Segundo trimestre de 2017. Rio de Janeiro: IBGE; 2017. [acessado 2017 Nov 8]. Disponível em: ftp://ftp.ibge.gov.br/Trabalho_e_Rendimento/Pesquisa_Nacional_por_Amostra_de_ Domicilios_continua/Trimestral/Fasciculos_Indicadores_IBGE/pnadc_201702_trimestre_caderno.pdf

11. Organização Internacional do Trabalho (OIT). Relatório mundial sobre proteção social 2014-2015: construindo a recuperação econômica, o desenvolvimento inclusive e a justiça social. Genebra, 2014. [acessado 2016 Ago 16]. Disponível em: http://www.ilo. org/secsoc

12. Majeed T, Forder PM, Tavener M, Vo K, Byles J. Work after age 65: a prospective study of Australian men and women. Australas J Ageing 2017; 36(2):158-164.

13. Boot CRL, Kruif ATCM, Shaw WS, Beek AJ, Deeg DJ, Abma T. Factors important for work participation among older workers with depression, cardiovascular disease, and osteoarthritis: a mixed method study. $J$ Occup Rehabil 2016; 26(2):160-172.

14. Moen P, Kojola E, Kelly EL, Karakaya Y. Men and women expecting to work longer: do changing work conditions matter? Work, aging and retirement 2016; 2(3):321-344. 
15. Fasbender U, Wang M, Voltmer JB, Deller J. The meaning of work for post-retirement employment decisions. Work, aging and retirement 2016; 2(1):12-23.

16. Castro CMS, Mambrini JVM, Sampaio RF, Macinko J, Lima-Costa MF. Aspectos sociodemográficos e de saúde associados ao trabalho remunerado em adultos (50-69 anos) na Região Metropolitana de Belo Horizonte, Minas Gerais, Brasil. Cad Saude Publica 2015; 31(8):1775-1787.

17. Hasselhorn HM, Apt W, organizadores. Federal Ministry of Labour and Social Affairs. Understanding employment participation of older workers: creating a knowledge base for future labour market challenges. Germany, 2015. [acessado 2016 Jul 29]. Disponível em: http://www.jp-demographic.eu/

18. Rosa SS. O perfil do idoso no mercado de trabalho brasileiro: análise das PNADs 2002 e 2012 [dissertação]. Rio Grande: FURG; 2015.

19. van Gameren E, Naranjo DV. Working and caring: the simultaneous decision of labor force participation and informal elderly and child support activities in Mexico. Lat Am J Econ 2015; 52(2):117-148.

20. Barbosa ALNH. Participação feminina na força de trabalho brasileira: evolução e determinantes. In: Camarano AA, organizadora. Novo regime demográfico: uma nova relação entre população e desenvolvimento? Rio de Janeiro: IPEA; 2014. p. 407-442.

21. West LA, Cole S, Goodkind D, Wan H. 65+ in the United States: 2010. U.S. Census Bureau. Washington; 2014.

22. Sala GA, Oliveira AHC. Labor force participation patterns and occupational segregation of older Argentineans and Brazilians. Apresentado no IUSSP 27th International Population Conference. Corea 2013. [acessado 2016 Ago 4]. Disponível em: http://iussp. org/en/event/17/programme/paper/3836

23. Pandey MK. Labor force participation among Indian Elderly: Does health matter? ASARC Working Paper. Institute of Economic Growth, India 2009.

24. Burdof A, van den Berg T, Avendano M, Kunst A, Mackenbach J. The effects of ill health on displacement from the labour market and potential impact of prevention. In: Börsch-Supan, organizador. First results from the survey of health, ageing and retirement in Europe (2004-2007). Mannheim Research Institute for the Economics of Aging, 2008. [acessado $2016 \mathrm{Jul}$ 29]. Disponível em: http://www.share-project.org

25. Pérez ER, Wajnman S, Oliveira AMHC. Análise dos determinantes da participação no mercado de trabalho dos idosos em São Paulo. Rev Bras Estud Popul 2006; 23(2):269-286.

26. Bós AMG, Bós AJG. A participação dos idosos gaúchos no mercado de trabalho e a força da relação renda/saúde. RBCEH 2004; 1(1):48-56.

27. Bruschini C, Lombardi MR, Mercado C, Bizzochi M. Base de dados sobre o trabalho das mulheres. Séries históricas, Fundação Carlos Chagas, São Paulo, 2010. [acessado 2016 Set 1]. Disponível em: http://www.fcc. org.br/bdmulheres/index.php
28. Damacena GN, Szwarcwald CL, Malta DC, Souza Júnior PRB, Vieira MLFP, Pereira CA, Neto OLM, Silva Júnior JB. O processo de desenvolvimento da Pesquisa Nacional de Saúde no Brasil, 2013. Epidemiol Serv Saúde 2015; 24(2):197-206

29. Souza-Júnior PRB, Freitas MPS, Antonaci GA, Szwarcwald CL. Desenho da amostra da Pesquisa Nacional de Saúde 2013. Epidemiol Serv Saúde 2015; 24(2):207-216.

30. World Bank. World Bank Group Gender \& Development. Washington, DC, 2014. [acessado 2016 Ago 16] Disponível em: http://www.worldbank.org/gender

31. World Bank. World development report: gender equality and development. Washington, DC, 2012 [acessado 2016 Jul 29]. Disponível em: https://siteresources.worldbank.org/INTWDR2012/Resources /7778105-1299699968583/7786210-1315 936222006/ Complete-Report.pdf

32. Instituto Brasileiro de Geografia e Estatística (IBGE). Sintese de Indicadores Sociais: Uma análise das condições de vida da população brasileira: 2017. Rio de Janeiro: IBGE; 2017. [acessado 2016 Fev 19]. Disponível em: https://biblioteca.ibge.gov.br/index.php/ biblioteca-catalogo?view $=$ detalhes\&id $=2101459$

33. Lima-Costa MF, Peixoto SV, Malta DC, Szwarcwald CL, Mambrini JVM. Cuidado informal e remunerado aos idosos no Brasil (Pesquisa Nacional de Saúde, 2013). Rev Saude Publica 2017; 51(Supl. 1):6s.

34. Taskila T, Shreeve V, Laghini M, Bevan S. Living long, working well: supporting older workers with health conditions to remain active at work. The Work Foundation, Londres, 2015. [acessado 2016 Ago 17]. Disponível em: http://www.theworkfoundation.com

35. Loeppke RR, Schill AL, Chosewwod C, Grosch JW, Allweiss P, Burton WN, Barnes-Farrell JL, Goetzel RZ, Heinen L, Hudson TW, Hymel P, Merchant J, Edington DW, Konicki DL, Larson PW. Advancing workplace health protection and promotion for an aging workforce. JOEM 2013; 55(5):500-506.

36. Camarano AA. O idoso brasileiro no mercado de trabalho. In: IPEA. Texto para discussão $n^{\circ} 830$. Rio de Janeiro: IPEA 2000. [acessado 2016 Ago 16]. Disponível em: http://www.ipea.gov.br

37. Headey B, Freebairn J, Warren D. Dynamics of mature age workforce participation: policy effects and continuing trends. Melbourne Institute of Applied Economic and Social Research. The Univervity of Melbourne 2010.

38. Jylha M. Whatis self-rated health and why does it predict mortality? Towards a unified conceptual model. Soc Sci Med 2009; 69(3):307-316.

39. Lima-Costa MF, Cesar CC, Chor D, Proietti FA. Self-rated health compared with objectively measured health status as a tool for mortality risk screening in older adults: 10-year follow-up of the Bambuí Cohort Study of Aging. AJE 2012; 175(3):228-235.

40. Szwarcwald CL, Souza-Júnior PRB, Esteves MAP, Damacena GN, Viacava F. Socio-demographic determinants of self-rated health in Brazil. Cad Saude Publica 2005; 21(Supl.):S54-S64. 
41. Barros MBA, Zanchetta LM, Moura EC, Malta DC. Autoavaliação da saúde e fatores associados, Brasil, 2006. Rev Saude Publica 2009; 43(Supl. 2):27-37.

42. Carrera-Fernandez J, Menezes WF. O idoso no mercado de trabalho: uma análise a partir da região metropolitan de Salvador. Rev. Econômica do Nordeste 2001; 32(1):52-67.

Artigo apresentado em 24/11/2017

Aprovado em 10/04/2018

Versão final apresentada em 12/04/2018 